\title{
Microscopical Characterization of Known Postmortem Root Bands Using Light and Scanning
}

\section{Electron Microscopy}

Jack Hietpas a, JoAnn Buscaglia ${ }^{\text {b }, \text { Adam H. Richard }}{ }^{\text {, Stephen Shaw }}{ }^{\text {d, Hilda S. Castillo }}{ }^{\text {, }}$, and Joseph

\section{Donfack $^{\mathrm{f}}$}

a Visiting Scientist Program, Counterterrorism and Forensic Science Research Unit, Federal Bureau of Investigation Laboratory Division, 2501 Investigation Parkway, Quantico 22135, Virginia, USA; jack.hietpas@ic.fbi.gov

b Counterterrorism and Forensic Science Research Unit, Federal Bureau of Investigation Laboratory Division, 2501 Investigation Parkway, Quantico 22135, Virginia, USA; joann.buscaglia@ic.fbi.gov

c Visiting Scientist Program, Counterterrorism and Forensic Science Research Unit, Federal Bureau of Investigation Laboratory Division, 2501 Investigation Parkway, Quantico 22135, Virginia, USA

d Trace Evidence Unit, Federal Bureau of Investigation Laboratory Division, 2501 Investigation Parkway, Quantico 22135, Virginia, USA; stephen.shaw@ic.fbi.gov

e Visiting Scientist Program, Counterterrorism and Forensic Science Research Unit, Federal Bureau of Investigation Laboratory Division, 2501 Investigation Parkway, Quantico 22135, Virginia, USA

${ }_{\mathrm{f} C o u n t e r t e r r o r i s m}$ and Forensic Science Research Unit, Federal Bureau of Investigation Laboratory Division, 2501 Investigation Parkway, Quantico 22135, Virginia, USA;joseph.donfack@ic.fbi.gov

Corresponding author:

JoAnn Buscaglia, $\mathrm{PhD}$

Counterterrorism and Forensic Science Research Unit

Federal Bureau of Investigation Laboratory

2501 Investigation Parkway

Quantico, Virginia 22135 USA

(703) 632-4553

(703) 632-4557 (fax)

joann.buscaglia@ic.fbi.gov

(C) 2016. This manuscript version is made available under the Elsevier user license 
Microscopical Characterization of Known Postmortem Root Bands Using Light and Scanning Electron Microscopy

\section{Abstract}

A postmortem root band (PMRB) is a distinct microscopic feature that is postulated to occur in hair remaining in the follicle during the postmortem interval [1]. The scientific validity of this premise has been highlighted in two recent high-profile criminal cases involving PMRBs $[2,3]$. To better understand the fundamental aspects of postmortem root banding, the microscopical properties of known PMRBs ${ }^{1}$ were characterized by light microscopy and scanning electron microscope (SEM) imaging of microtomed sections of hairs showing root banding. The results from this study show that the appearance of the PMRB may be due to the degradation of the chemically labile, non-keratin intermacrofibrillar matrix (IMM) in the pre-keratin region of anagen hairs. In addition, this degradation is confined to the cortex of the hair, with no apparent damage to the layers of the cuticle. These results could provide valuable information for determining the mechanism of band formation, as well as identify a set of microscopical features that could be used to distinguish hairs with known PMRBs from similarly looking environmentally degraded hairs.

\section{Introduction}

\footnotetext{
${ }^{1}$ In this paper, a known PMRB is defined as a dark elliptical band in a hair (when viewed in transmitted light) in the pre-keratin region of the hair root and is also confirmed to have been recovered from the tissue of a deceased person during the postmortem interval. This is equivalent to a Stage 2 band as defined by [4] Koch SL, Michaud AL, Mikell CE. Taphonomy of Hair-A study of postmortem root banding. J Forensic Sci. 2013:s52-s9, [4] ibid.
} 
21 ancestry, disease, damage, growth stage, and color alteration[6]. Observations made using light

22 microscopy have also identified distinct root characteristics that have been interpreted to be indicative

23 of the hair being shed from a deceased person[7]. These characteristics have been termed "dead man's

24 root," "dead man's ring," "putrid root" or "post mortem root band (PMRB);" the later term is used

25 throughout this paper. A PMRB is defined by the Scientific Working Group for Materials Analysis

26 (SWGMAT) as "the appearance of an opaque microscopic band near the root area of hairs from a

27 decomposing body" [8]. Microscopic hair features (e.g., PMRB (Fig. 1)) that reliably indicate the hair

28 donor was deceased may provide valuable information for reconstruction purposes that require making

29 meaningful associations between people and/or locations with a known sample from a deceased person

30 [9]. However, the scientific validity of whether or not a hair showing the characteristics of a PMRB can

31 be positively identified as deriving from a deceased person is still under question [2, 3, 10], largely due

32 to the lack of a definitive mechanism for PMRB formation.

This paper focuses on the ultrastructural characterization of PMRBs using microscopical

34 techniques, which may provide fundamental information for defining the potential mechanism(s) of

35 band formation. The second paper of this series will address aspects of the mechanism of PMRB

36 formation. Before the details of PMRB characteristics are discussed, a detailed review of human hair

37 biochemistry, structure, and growth is presented. A strong foundation in these topics is required for

38 meaningful interpretations of the microscopical and ultrastructural observations of hairs that display

39 PMRBs.

40

\section{The Biochemistry of Human Hair}

Human hair is a multicellular protein fiber composed of several morphological units, each having

43 a specific chemical composition [11]. The hair fiber is composed primarily of protein (keratin and 
44 keratin-associated proteins) and lipids. Proteomic studies have identified $\sim 340$ proteins in samples of

45 human head hair $[12,13]$. Extensive disulfide bonding between thiol functional groups (-R-SH) present

46 in the amino acid cysteine (thus forming cystine following disulphide bonding) is the reason for hair's

47 significant resistance to physical and chemical degradation [11].

Hair Structure

The complex structure of hair has been elucidated in several fundamental studies that relied on

51 high-resolution transmission electron microscopy [14-19]. The hair and associated follicle consists of

52 several distinct structural layers including the external root sheath and internal root sheaths, cuticle,

53 cortex and medulla (Fig. 2). Each of these units will be discussed in detail below.

The Root Sheaths

The function of the outer root sheath is not definitively known, but it may contribute to shaping

56 the hair fiber [20]. Surrounding the hair is the inner root sheath, which is composed of three concentric

57 layers: the inner root sheath cuticle, the Huxley layer, and the Henley layer [21]. All three layers are fully

58 keratinized "soft" keratin (e.g., stratum corneum) [21]. This type of keratin differs from "hard" keratin

59 (e.g., hair, nails, and beaks) in having a lower cysteine content (2.6\% vs. $7.9 \%$, respectively) [22]. The

60 inner root sheath is truncated at the point where the sebaceous duct intersects the hair shaft [21]; it is

61 thought that proteases secreted in the sebaceous duct are responsible for limiting the height of the root

62 sheath [21]. The outer root sheath extends from the lower end of the hair bulb to the entrance of the

63 sebaceous duct where to changes into epidermis; at this location, the outer root sheath becomes

64 keratinized [21]. 
The cuticle has a layered structure (Fig. 3) composed of overlapping scales [23, 24]. In addition,

67 the individual scales also have a layered structure composed of four major units, each differing in disulphide and isopeptide bonding [11]. The epicuticle is a hydrophobic layer approximately $5 \mathrm{~nm}$ thick that is present on the outer surface of the scale. The next layer, called the " $\alpha$-layer," is keratinous and contains a very high amount of sulfur [24]; the covalent disulphide bonds give this layer resistance to physical and chemical attack [11]. The third layer, the exocuticle, also contains a high amount of sulfur

72 ( 11\%) [25]; thus, this layer (through disulphide bonding) provides physical and mechanical resistance

73

and has been shown to be resistant to proteolytic digestions [19]. The inner most layer, the endocuticle, is non-keratinous and consists mainly of protein and cellular debris. It contains very little cysteine/cystine but large amounts of free acidic and basic groups [24]. Swift and Bews [19] reported that the endocuticle is solubilized by proteolytic enzymes; this layer is hydrophilic and consequently swells in water [23]. The cell membrane complex (CMC), which contains very little protein and is composed of polysaccharides and lipids, functions to cement the cuticle layers together [24].

\section{The Cortex}

The cortex accounts for the bulk of the hair fiber weight; it contains fibrous and matrix proteins, which are responsible for the mechanical properties of the hair fiber [14]. The cortex is composed of CMC and three cortical cell types: ortho-, para- and meso-cortex cells, each differing in their crystallinity [11]. The cortical cells are spindle-shaped and are approximately $100 \mu \mathrm{m}$ long and $25 \mu \mathrm{m}$ wide [25]. These cells contain bundled aggregates of macrofibers that are embedded in a non-keratin material, termed the intermacrofibrillar matrix (IMM) (Fig. 3). A cortical cell typically contains 5-8 macrofibers, each with a diameter of $300-500 \mathrm{~nm}$ [11]. The IMM accounts for 1-3\% of the fiber weight [26] and its composition is a complex mixture of protein and organelles leftover from the keratinization process. 
More specifically, the IMM is primarily composed of nuclear and cytoplasmic remnants, endoplasmic

90 reticula, Golgi apparati, and mitochondria, among other minor cellular components [11]. The IMM is one of the three non-keratinous components of hair (the CMC and endocuticle being the other two

92 [26]). Like the endocuticle and CMC, there is little cysteine/ cystine present in the IMM and the region is

93 hydrophilic, consequently swelling in aqueous solutions. In addition, the IMM, along with the CMC and 94 endocuticle is a pathway for aqueous solutions and components (e.g., hair dyes) to enter the cortex $95[26]$.

97 composed of keratin-associated proteins $[11,14]$. Each microfibril is composed of 7-8 single or paired 98 protofilaments; the subunit of a protofilament is a four-chain $\alpha$-helix structure [11].

The Medulla

The medulla, which is contained within the cortex, consists of hollow cells with a skeleton of 102 amorphous proteins and fine filaments [11]. The medulla is typically filled with gas, thus the region commonly appears dark in transmitted light microscopy. The function and formation of the medulla is not well understood; however, it does not contribute to the mechanical or cosmetic properties of 105 human hair [27].

\section{Hair Growth}

Hair growth can be divided into three major phases. Anagen is the active growth phase during which follicle development occurs and the hair shaft is formed. Approximately $80-95 \%$ of all human head hairs are in the growing phase at any one time [28]. The anagen phase can vary from 2 to 6 years, 
111 and an approximate value for the rate of growth is $400 \mu \mathrm{m} /$ day [25]. At the end of the anagen phase,

112 the hair enters the catagen phase, which lasts approximately 1-2 weeks. During this phase, the follicle

113 slowly begins programmed cell death (apoptosis). The telogen phase follows the catagen phase and

114 normally lasts about 2 to 3 months [29]. During this time the hair does not grow but stays attached to

115 the follicle while the dermal papilla stays in a resting phase. Approximately $5-15 \%$ of all hairs are in the 116 telogen phase at any one time. The hair is still held in the skin through friction, but can be shed (exogen

117 phase) through mechanical abrasion or pushed out by an underlying actively growing (anagen phase)

118 hair follicle. Most people naturally shed approximately 50-150 scalp hairs per day [29, 30].

\section{PMRBs: Conditions for and Progression of Formation}

A review of the literature pertaining to the conditions in which PMRBs form notes several

122 common observations. First, PMRBs have been reported primarily in anagen-phase hairs [1, 4, 31].

123 However, Linch and Prahlow [31] and Koch et al. [4] observed PMRBs in catagen phase hairs. No PMRBs

124 have been observed in hairs that are in the telogen phase [1,31]. Consequently, empirical data suggest

125 that hair must be in either the active growing phase (anagen) or early stages of quiescence (catagen)

126 prior to an individual's death to be susceptible to PMRB formation. Given this apparent growth cycle

127 constraint, human hair from the scalp (large proportion of anagen growth phase) is the most common

128 type a hair to display a PMRB. Hair from other somatic origins (e.g. pubic, limb, body) are more likely to

129 be in the telogen phase and thus not susceptible to PMRB formation.

The second reported observation is that the hair must be in the skin while the body is

131 decomposing for a hair to display root banding [32]. Studies have been conducted to attempt to

132 replicate PMRBs by exposing hairs to various environmental factors [32-34]. Although some of these

133 studies have concluded that some PMRB characteristics could be produced by environmental factors, 
none have proven to replicate characteristics that an experienced hair examiner would identify as

135 PMRBs. The primary differences in characteristics between hair that is environmentally degraded and hair displaying a true PMRB are the morphology and location of root degradation.

The final factor influencing PMRB formation is the length of the postmortem interval (PMI). The

138 time observed for the onset of a PMRB varies widely [10] from as little as 8 hours [1] to "a minimum of 7 139 days" (expert witness testimony [3]). Approximately one week is a commonly cited PMI for PMRB 140 formation, which is supported by Koch et al. [4]. In addition to these three factors, Koch et al. [4] and

141 Collins [32] reported a correlation between temperature and occurrence of PMRBs; an increase in 142 temperature at the location of cadaver decomposition decreases the time for the occurrence of PMRBs.

145 Linch et al. [35] reported that following the formation of a typical PMRB, the band further develops 146 (inferred continued degradation to the hair) to produce either a "brushy end," where the proximal end 147 is highly frayed, or a "hard point," where the soft keratin of the hair is completely removed leaving 148 behind the more resistant hard (keratinized) region of the hair shaft. Koch et al. [4] presented a similar 149 PMRB development scheme; however, they define four major stages. Stage 0 hair from a deceased 150 person shows no observable differences in the characteristics of the hair roots from antemortem hair 151 samples. Hair in stage 1 shows some limited darkening of the pre-keratin region of the hair root. Hair in 152 stage 2 shows the typical dark band that is confined to the pre-keratin region of hair (i.e., the "typical" 153 PMRB). Finally, stage 3 (last phase of hair decomposition) hair has a "brushy" or "hard point" proximal 154 end [1, 4, 31]. In addition, both Koch et al. [4] and Linch and Prahlow [31] have reported that the hair 155 shaft darkens in the root region during PMRB formation. 
It is also important to note that the abundance of hairs from a cadaver that display stage 2

157 PMRBs is highly variable. Based on the data from 21 cadavers, the average percentage of hairs

158 displaying stage 2 bands is $5 \pm 9 \%(1 \sigma)$ with a range of $0-31 \%$ [4]. However, $99 \%$ of the hairs from one

159 additional test cadaver (that was buried in soil for 3 years) displayed stage 2 PMRBs [4]. These data may

160 suggest that the conditions for PMRB formation are quite strict. Experiments to investigate potential

161 mechanisms/ conditions for PMRB formation will be the focus of the second paper of this research.

162

\section{Scientific Validity that a PMRB is an Indicator of Derivation from a Deceased Individual}

The scientific validity of PMRBs has received considerable attention. In the case of People v. Kogut a hair displaying a PMRB, and microscopically consistent with hair from a deceased victim, was found in the trunk of the defendant's vehicle [3]. The finding of the victim's hair (which reportedly displayed a PMRB) in the vehicle was argued to be from either police negligence or misconduct. These interpretations were based on the appearance of the band and the short postmortem interval (on the order of a few hours). The United States District Court, Eastern District of New York held a Daubert hearing [36] to determine the validity of the PMRB evidence; the court's ruling allowed testimony concerning PMRBs to be included at the subsequent wrongful conviction case.

Postmortem hair root banding was a significant piece of evidence in the high-profile "Casey Anthony Case" [2]. A single PMRB hair that was microscopically consistent with the victim was found in the trunk of the defendant's vehicle. A Frye hearing [37] was held with the defense challenging the scientific validity of PMRB evidence. The defense argued that experts do not know the exact or potential causes of root banding other than death, and that science leaves too much interpretation to the forensic hair examiner. The Frye hearing to exclude the testimony concerning PMRB was denied. During trial testimony, Federal Bureau of Investigation's hair examiner correctly stated [we] "cannot assert that characteristics of decomposition seen in hairs are due to them being shed from a deceased individual." 
179 However, the examiner also stated "to date, no research projects have been able to replicate the 180 characteristics of postmortem rooting banding, but we don't know that nothing else can cause those 181 characteristics."

Several research projects have been undertaken to assess the potential to induce PMRB characteristics in antemortem hairs via exposure to various environmental conditions (e.g., soil, water

184 immersion, vehicle trunk), thus testing the premise that hairs with root bands are solely derived from 185 deceased individuals [33, 34, 38, 39]. Delgado [33] and Domzalski [34] cautioned that some antemortem 186 hairs subjected to different environmental conditions could be falsely identified as hair containing a 187 PMRB, but also acknowledged that additional studies comparing environmentally exposed antemortem 188 hairs to postmortem hairs should be conducted. A blind-study by Shaw 2012 [39] showed that hair examiners correctly identified known PMRBs (true positive) for $99.5 \%$ of the evaluated samples; no errors occurred when quality assurance practices requiring verification by a second examiner were

191 included. In addition, examiners correctly differentiated environmentally degraded antemortem hairs 192 (true negative) from hairs containing known PMRBs.

To help shed light on the issues presented above, this research was focused on better understanding PMRBs at the light microscopical and ultrastructural spatial scales. A detailed 195 understanding of the physical characteristics that define a PMRB will provide vital information for 196 shedding light on potential mechanisms of band formation and may also provide metrics to differentiate 197 true PMRBs from environmentally degraded antemortem hair.

Methods

Individual hairs $(\mathrm{n} \approx 250$ hairs from eight donors) displaying known PMRBs were obtained from the Koch et al. study [4]; the determination of known PMRB was a consensus of several qualified hair 201 examiners and by this research team. The Koch et al. study involved placing cadavers in different 


\section{Results} that a PMRB occurs approximately $0.5 \mathrm{~mm}$ distal to the root tip and $2 \mathrm{~mm}$ from the skin surface; our 224 findings are consistent with these reported values. In addition, using image analysis, we measured the

Approximately 15 hair samples (from 3 donors) displaying stage 1 or stage 2 banding (as defined in the Koch et al. study [4]) were selected for sectioning and detailed examination using scanning electron microscopy (SEM). Hair samples were removed from the slide preparations using xylene to dissolve the Permount. The hair samples were then dehydrated in 5 minute steps using a graded series of ethanol solutions $(50 \%, 70 \%, 90 \%$, and $95 \%)$. After dehydration, hair samples were embedded in either Buehler Epo-thin ${ }^{\circledR}$ or Electron Microscopy Sciences Embed-812 ${ }^{\circledR}$. Hair samples were subsequently sectioned using a Leica EM UC6 ultramicrotome with a histology diamond knife. Both longitudinal and cross-sections of 0.5-3 $\mu \mathrm{m}$ thickness were gold-coated and examined using a Zeiss-Leo 1450VP SEM.

Light Microscopy

A PMRB is a dark band when viewed in transmitted light; however, it can also appear white in dark field or reflected illumination (Fig. 1). The band consists of a complex network of interwoven elongated elliptical void spaces that are oriented parallel to the hair shaft (Fig. 4). The quantitative microscopical characterization of known PMRBs was first reported by Petraco et al. [1]. They reported 
length of stage 2 and 3 bands (with intact proximal ends) in 220 hair samples from eight cadavers. The average length was determined to be $252 \mu \mathrm{m}+/-48 \mu \mathrm{m}(1 \sigma)$.

The overall morphology of the band is broadly elliptical. At the proximal end, the transition from nondegraded hair to the band is abrupt (Figs. 1, 5-7); however, at the distal end, the transition from the banded region to the nondegraded portion of the hair is more gradual or diffuse (Figs. 1, 5-7). The banded region is constrained to the cortex of the hair and there is no observable damage to the cuticle (Figs. 5-6). The band generally develops such that it affects all available space contained within the cuticle, thus the width of the band is typically that of the hair shaft. In some instances, the banded region is slightly bulged outward, giving the hair a swollen appearance in the banded region (Fig. 5). This observation may be due to one or more of the following: 1) the region of the hair is coincidently slightly wider than the rest of the hair shaft; 2 ) the degradation that results in PMRBs causes internal expansion; or 3) the structural integrity of the hair is compromised in this region, allowing the area to expand slightly during sample handling and mounting for microscopy.

In addition, the most proximal end of the band (hypothesized start of the band) coincides with the region where the cuticle is first discernible (Fig. 5). In this study, no known PMRBs were observed to extend significantly below this region, and this provides a good reference point for determining a PMRB in a hair of unknown origin. It was also observed that in several instances, hairs displaying PMRB in stages 1 or 2 also contained intact "soft" keratin root sheaths (Fig. 7). This finding may have important implications for understanding the mechanism of PMRB formation and will be further developed in the next paper of this series.

\section{Scanning Electron Microscopy}

Microtomed longitudinal sections were examined at 100-50,000x magnification. The accelerating voltage was kept at a moderate range of 5-15 keV to enhance spatial resolution. The 
location of PMRB damage is readily apparent (Fig. 8). Consistent with light microscopy observations, the "banded" region consists of a complex collection of void spaces. These voids are roughly elliptical in shape, with their major axis parallel to the hair fiber length. Although the cortex of a normal, healthy hair may contain small void spaces (e.g., cortical fusi), the morphology and the extreme high density of the spaces in the PMRB are significantly different (compare Fig. 2 to Figs. 8-10). This is further supported by TEM-scale ultrastructural studies from the cosmetics and textiles industries, which do not report large networks of void spaces in normal healthy hair [14, 17, 25, 40-42].

The $200-600 \mathrm{~nm}$ fibers that encompass the void spaces are interpreted to be macrofibers. The IMM that binds macrofibers to one another appears to be preferentially degraded in the PMRB region. However, we cannot exclude additional degradation to the cellular wall of the cortical cells that contain the macrofibers; to make this assessment, higher resolution transmission electron microscopy (TEM) would be needed. Apparent remnants of the IMM can be seen as the jagged "saw-tooth" patterns between the separated macrofibers (Fig. 9). The morphology of these structures may suggest a mechanical "pull-apart" method of degradation (this is further addressed in the Discussion). There appears to be insignificant cross-fiber (perpendicular to the hair length) degradation. The damage is oriented parallel to the hair length, effectively "unzipping" the macrofibers from one another. In addition, the void spaces are present in several stages of band development. In the center of the "band," the void spaces are well developed with a significant amount of IMM degradation. However, the distal and, to a lesser extent, the proximal ends of the "banded" region show partially opened void spaces (i.e., less degradation of the IMM) (Fig. 9).

The degradation mechanism that is responsible for PMRBs does not appear to affect the cuticle (Figs. 5, 6, 10). The "band" shows a distinct and abrupt termination at the interface with the cuticle. At 
271 the cuticle was observed (Fig. 10).

273 scattered throughout the cortex of the hair and the cuticle layers are intact, consistent with the findings

274 from the longitudinal sections. In addition, high-magnification examination of the cut ends of the

275 individual macrofibers does not show indication of degradation to the microfibers. However,

276 verification of this finding would require using higher magnification transmission or field-emission

277 electron microscopy.

278 Discussion

279 The results from this study show that the characteristics of a PMRB may be due to the preferential 280 degradation of the IMM within the pre-keratin/ keratogenous region of anagen and possibly catagen

281 hairs. The reason for the apparent localized degradation to the IMM is likely due to its hydrophilicity;

282 the region is wet (water is liberated from the biosynthesis of proteins), and the region is not fully

283 keratinized [43]. During the anagen phase, the cortical cells above the pre-keratin/ keratogenous zone

284 become stabilized due to coalescence, consolidation, and hardening; these processes are facilitated by

285 cortical cell dehydration, hydrogen bond formation, and disulphide bonding [44]. These cellular

286 modifications are hypothesized to limit the distal extent of the PMRB because the mature cortex is more

287 resistant to degradation (empirically shown in the study by Chang et al. [45]). In addition, we

288 hypothesize that the PMRB does not extend below the pre-keratin/ keratogenous zone because the

289 IMM (material leftover from the keratinization process) is not present, and thus not available for

290 degradation. 
perpendicular to the hair shaft (Figs. 4, 8-9), the absence of observable damage to the endocuticle or cuticle CMC layers (Fig. 10), and no burrowed fungal pathways through the cuticle [46]. There is also no evidence for external microbes entering the hair shaft either through the cuticle or from the soft root tip. This is consistent with the observation that many hairs with complete stage 2 PMRBs have fully intact root sheaths, which are made of labile "soft" keratin (Fig. 7). The root sheaths are a readily accessible, nutrient-rich material that bacteria would assuredly digest prior to attacking the IMM.

The style of degradation to IMM may provide clues to potential causative agents for PMRB formation. The appearance of the void spaces does not show transverse directionality (e.g. progression inwards or outwards, nor from one side of the hair shaft to the other). This suggests that the causative agents that lead to PMRBs are not degrading through the cuticle as they penetrate the hair shaft. However, the band is more fully developed at the proximal end (near the start of the cuticle) than at the distal end; this could suggest distal propagation parallel to the hair shaft.

The isolated, partially opened void spaces that are observed in both the distal and proximal ends of the PMRB are interpreted to be the result of incomplete degradation of the IMM and/or failure of the void spaces to connect to the central mass of degradation (the "band"). In addition, the vulnerability of the IMM may vary depending on the localized degrees of hydration and keratinization in the distal region of the "band". Thus, some portions of the pre-keratin/keratinagenous region can be degraded, while others are appear unaffected; this may explain the diffuse nature of the transition from "band" to apparently unaffected cortex (Figs. 4-9). On the basis of the observed degradation to the IMM, both chemical and mechanical attacks are viable mechanisms for PMRB formation. The "pull-apart" structures in Fig. 9 may suggest mechanical degradation. The IMM appears to be stretched transversely to the point to where it is eventually ruptured, thus producing the characteristic "saw tooth" patterns. One possible origin for this apparent mechanical damage would be the trapping and build-up of 
decomposition gases within the pre-keratin region. However, chemical or enzymatic attack to the IMM

317 or follicle components is also possible; such causative agents may be components secreted by bacteria,

318 released during autolysis, or perhaps unregulated biological pathway processes (e.g., proteases, other

319 enzymes, $\mathrm{pH}$ changes, or other water soluble components). All these phenomena, and others, might

320 occur. Experiments designed to test the efficacy of these potential causative agents in the formation of

321 PMRBs are the focus of the next paper in this series.

\section{Conclusions}

The results from this study show that the manifestation of postmortem root banding is the

324 result of the apparent preferential degradation of the intermacrofibrillar matrix (IMM) that cements

325 together adjacent macrofiber bundles. The degradation of this material facilitates the development of

326 elliptical-shaped void spaces that are confined to the pre-keratin region of the cortex of the hair. No

327 significant damage was observed to the layers of the cuticle. In addition, the proximal end of the PMRB

328 coincides well with the location where the cuticle layer of the hair is first recognized as a distinct

329 structure.

This study provides valuable descriptive and quantitative information that could be used in assessing whether a questioned hair contains a PMRB. In addition, the results reported may provide a foundation from which to differentiate environmentally degraded hairs that display characteristics similar to those of hairs with true PMRBs. In the next papers of this series, known PMRBs will be examined using high-resolution TEM analysis to further refine the identity of the IMM component(s) (e.g., nuclear remnant, cellular remnants) as well as to assess potential degradation of the cortical cell information concerning PMRB formation. 

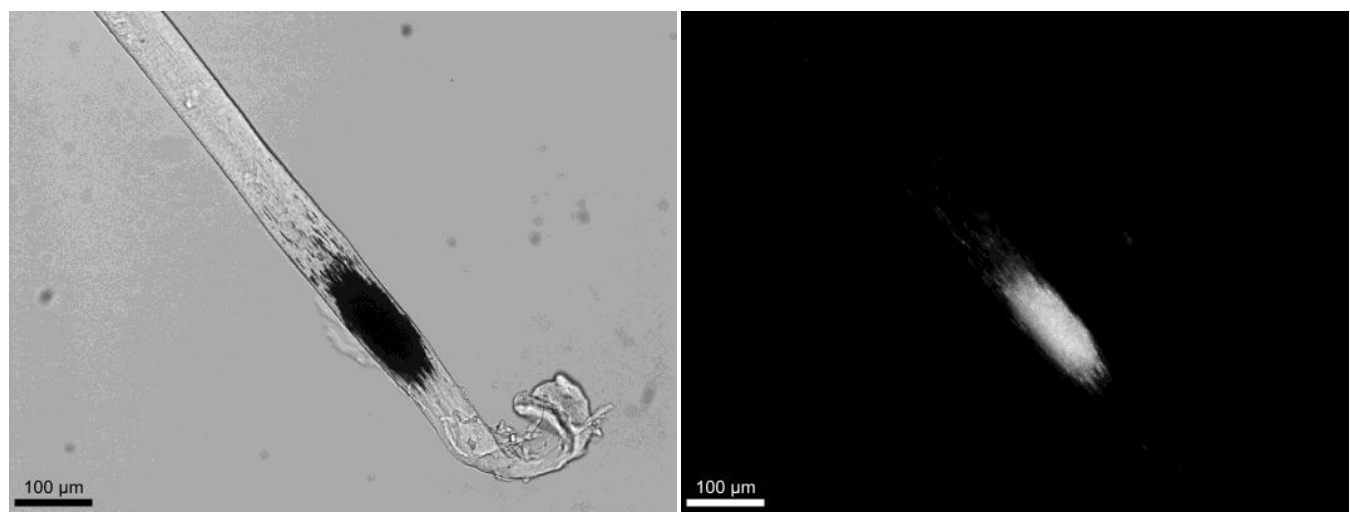

Figure 1. Photomicrographs of a hair displaying a known postmortem root band in transmitted (left) and oblique (right) illumination. The difference in appearance suggests that the "band" is a collection of air (gas) pockets [1].

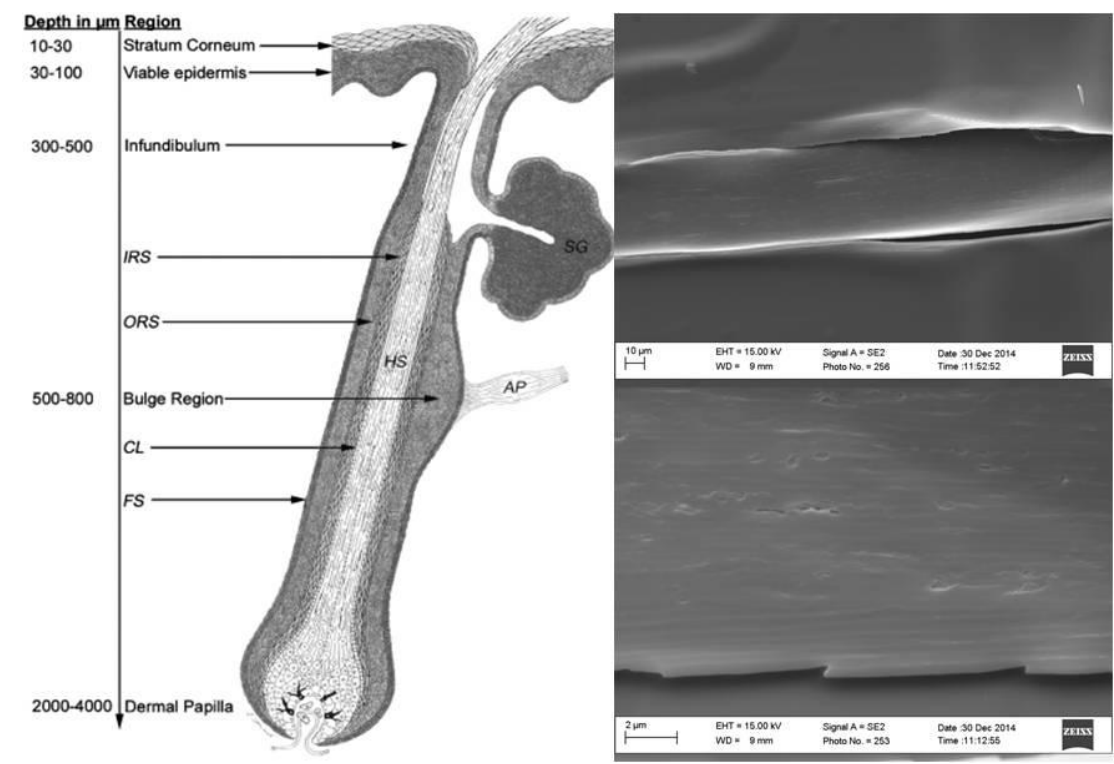

Figure 2. Schematic illustration of a hair follicle and SEM images of longitudinal sections of a normal antemortem head hair. IRS: inner root sheath, ORS: outer root sheath, CL: cuticle layer, FS: follicle sheath, SG: sebaceous gland, AP: arrector pili. Schematic illustration used with permission from Karger Publishing [47]. 


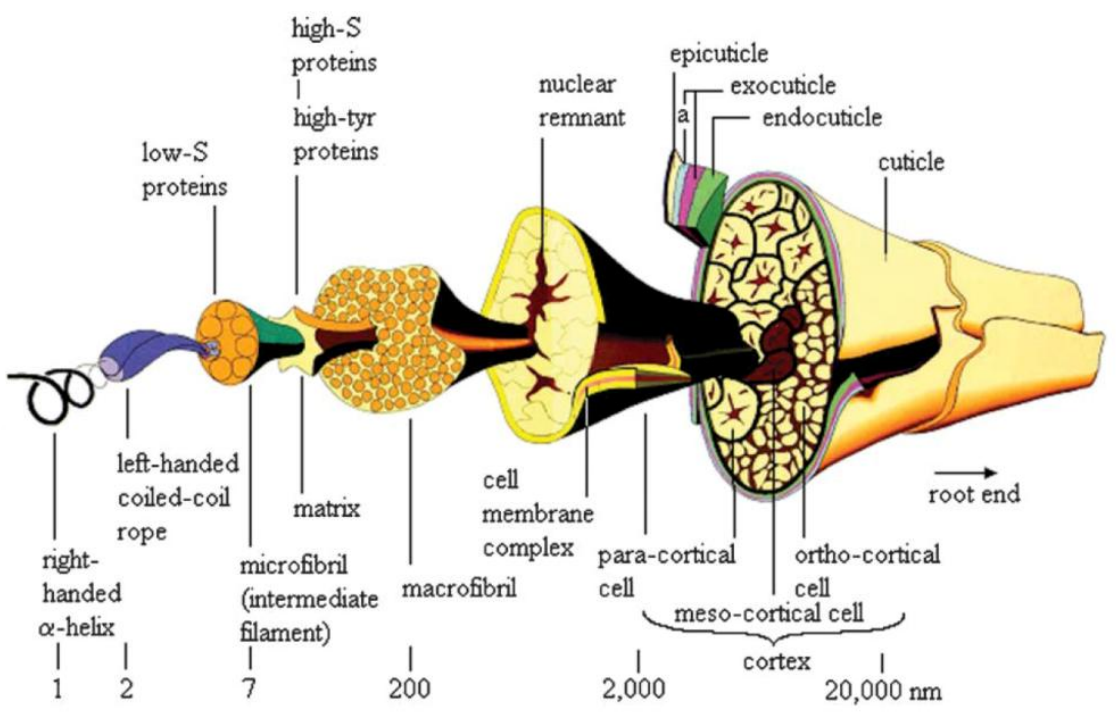

354 Figure 3. Schematic illustration showing the complex ultrastructure of hair. Note that the macrofibers are held to each other by the intermacrofibrillar matrix (IMM). Image used with permission from J. Wiley and Sons [11].

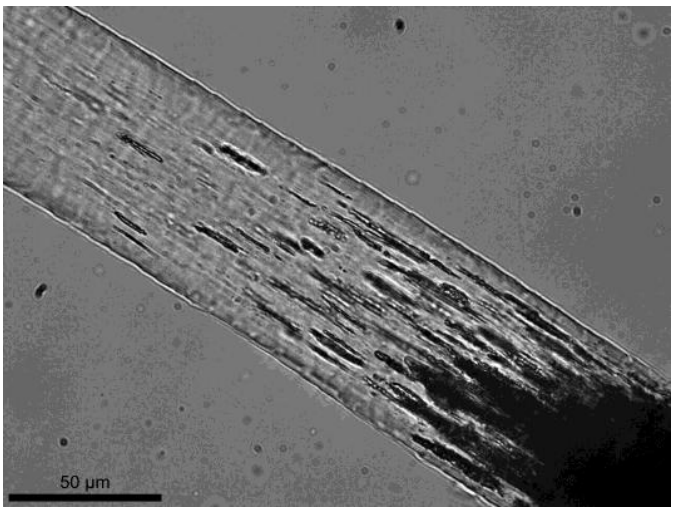

Figure 4. Photomicrograph of the distal end of a PMRB. Note that the elongated voids are not necessarily inter-connected and that they are oriented parallel to the hair fiber length. 

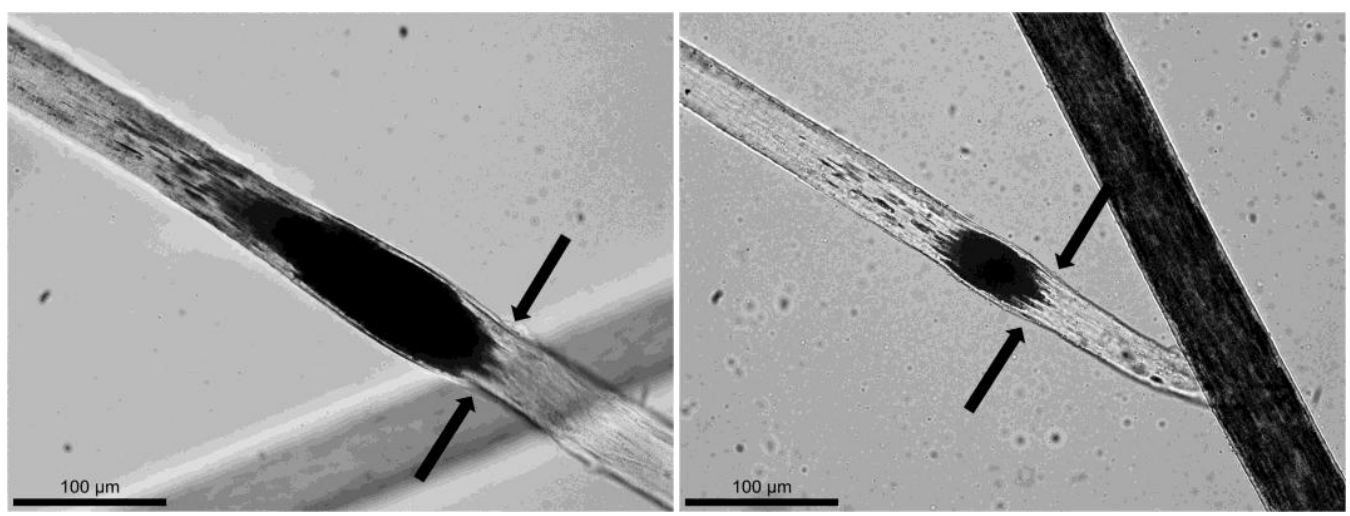

Figure 5. Photomicrographs of hair displaying known PMRBs. Note the intact cuticle that surrounds the "band". In addition, note that the most proximal end of the "band" starts very close to where the cuticle is first discernible.
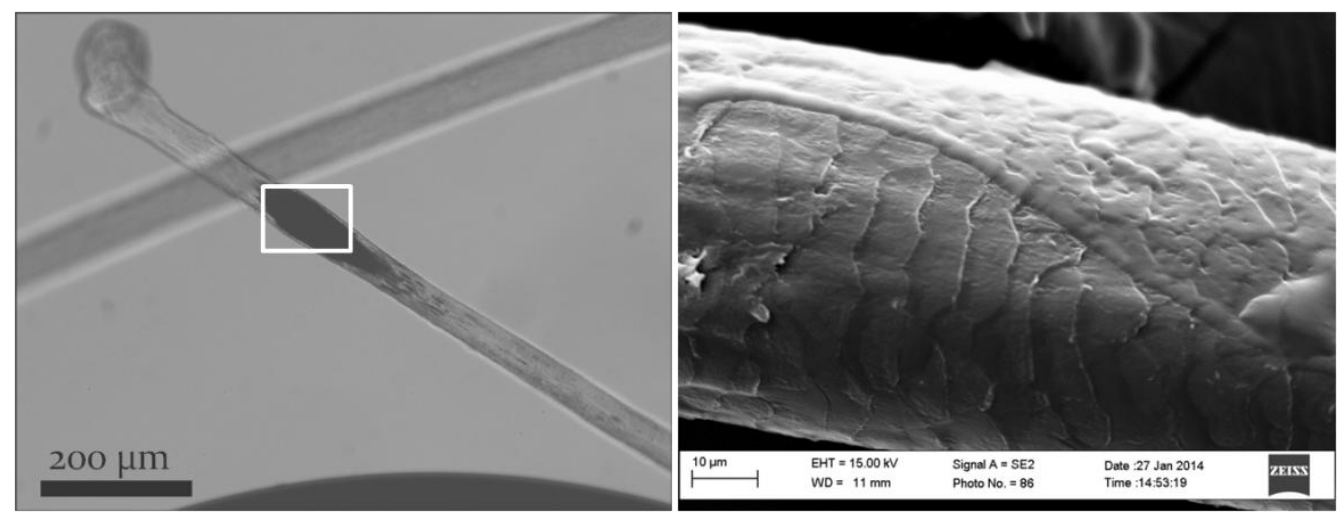

Figure 6. Light micrograph of a hair displaying a known PMRB (left) and corresponding SEM image (right) of the external cuticle surface at the location of the band (white rectangle). Note there is no discernible degradation to the external surface of the cuticle. However, the cuticle can display a "curtain-like" appearance with advanced stages of hair decomposition. 

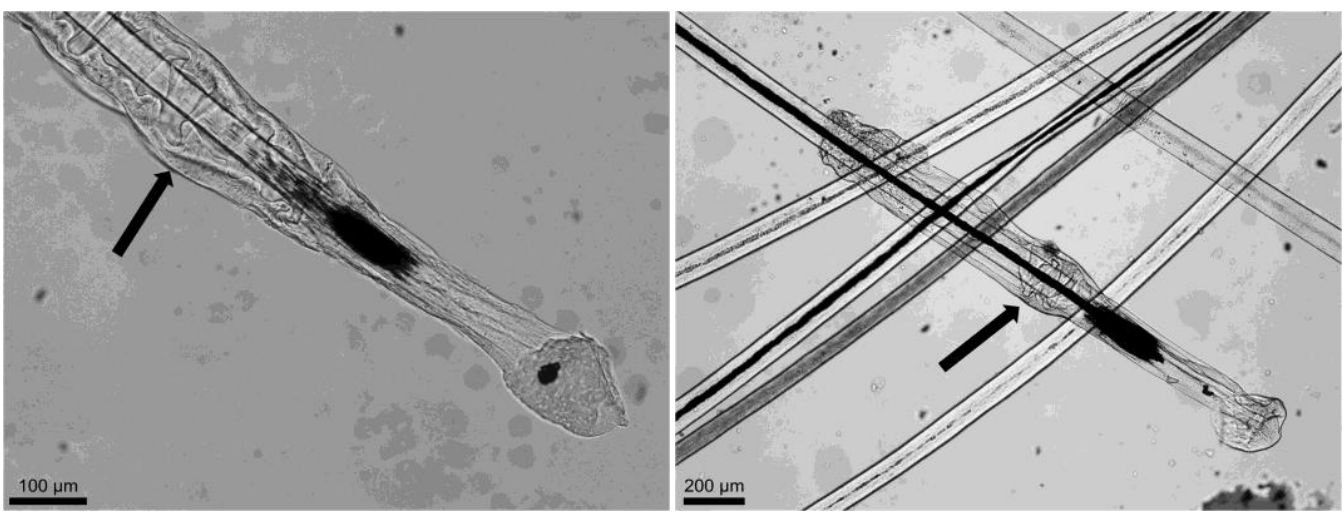

Figure 7. Photomicrographs of two hairs displaying known full stage 2 PMRBs. Note the intact condition of the soft keratin root sheaths present. It is common, though not required, for a hair that displays a PMRB to also have a root sheath.

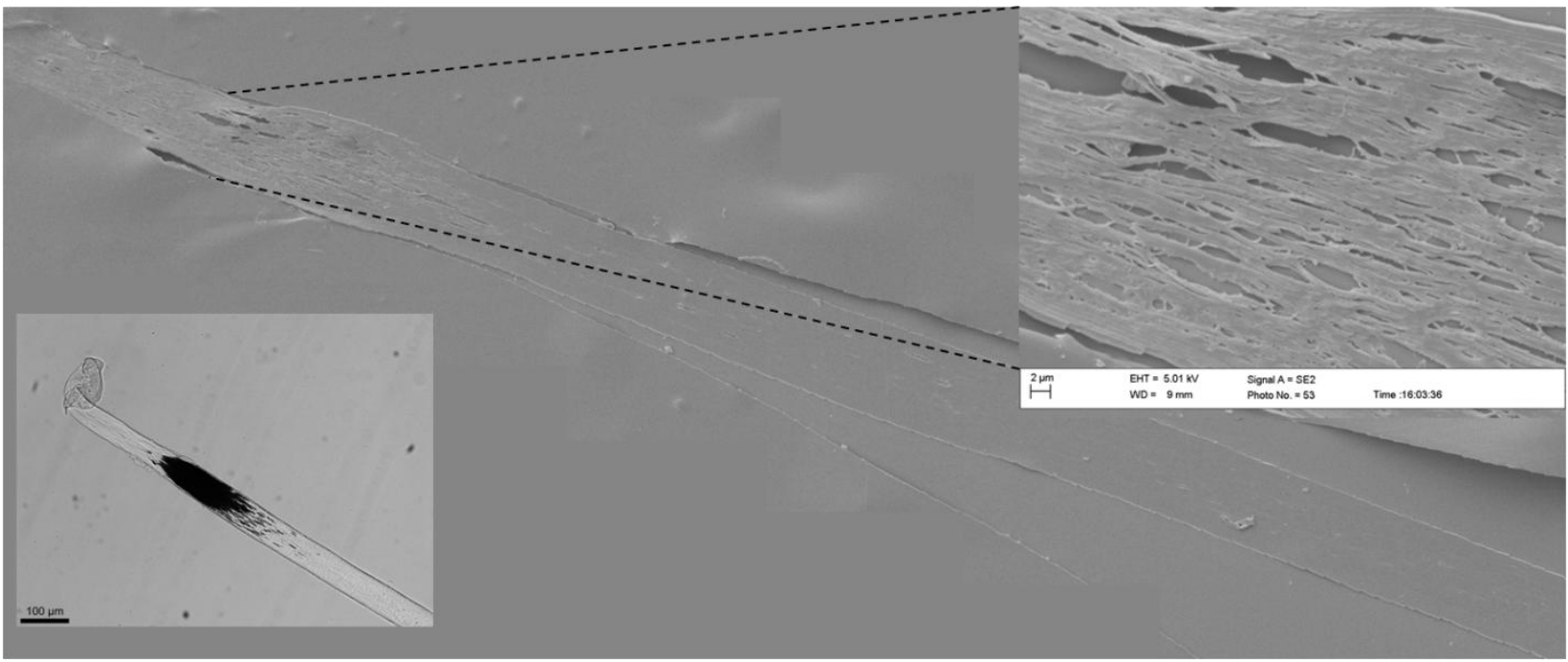

Figure 8. SEM image map of ultramicrotomed longitudinal section of a hair displaying a known PMRB. Note that the epoxy resin has separated from the hair section. Left inset: light micrograph of the sectioned hair. Right inset: note the significant degradation parallel to the length of the hair fiber. Compare extensive damage observed in the PMRB with the same region of a healthy antemortem hair in Fig. 2. 

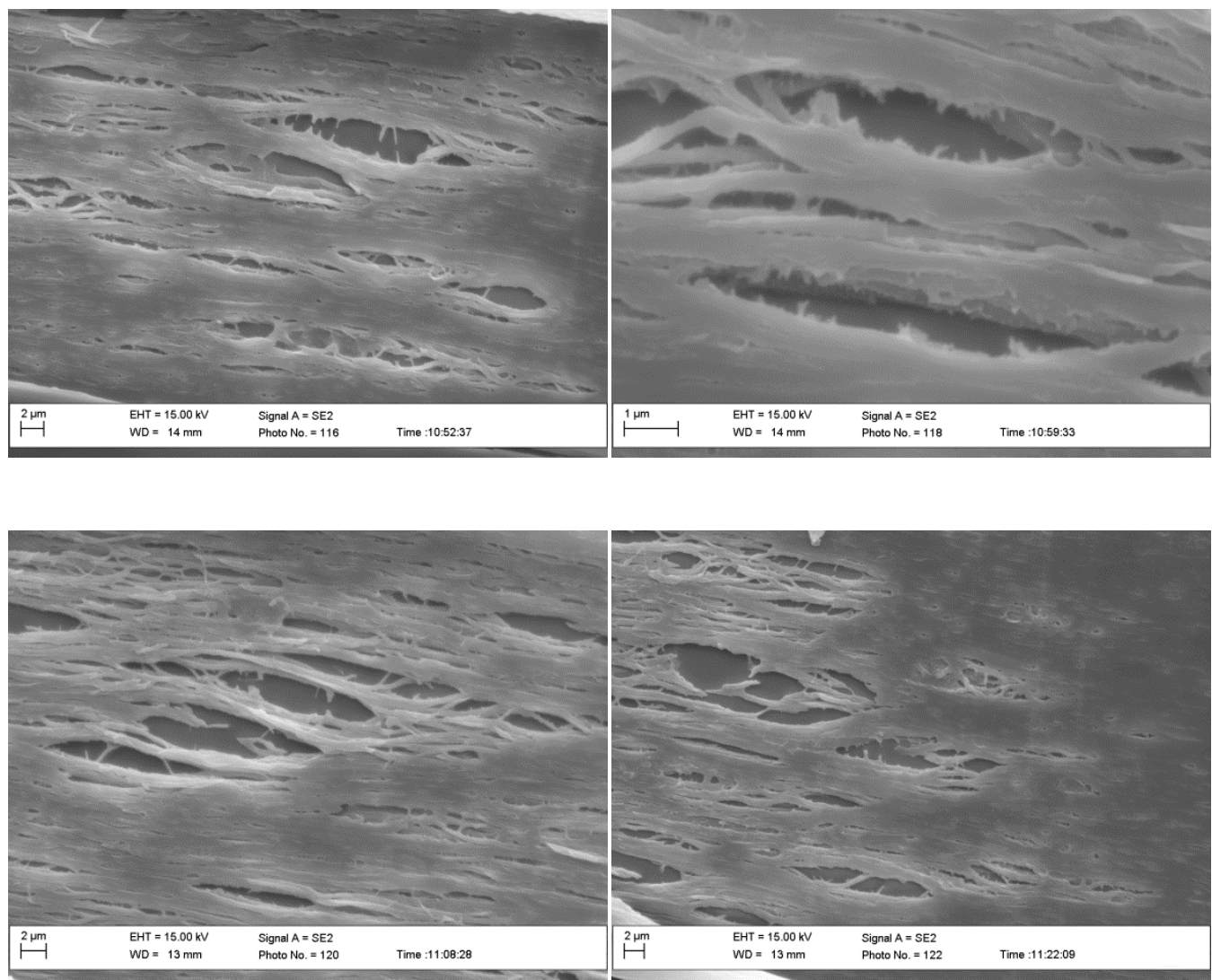

Figure 9. SEM images of longitudinal sections of the "banded" region from a hair displaying a known PMRB (same hair shown in Fig. 1). The top two images show the characteristic "saw-teeth" pattern of apparent remnant IMM. The bottom left image was taken from the midpoint of the banded area, and the bottom right one from the distal end of the banded region.

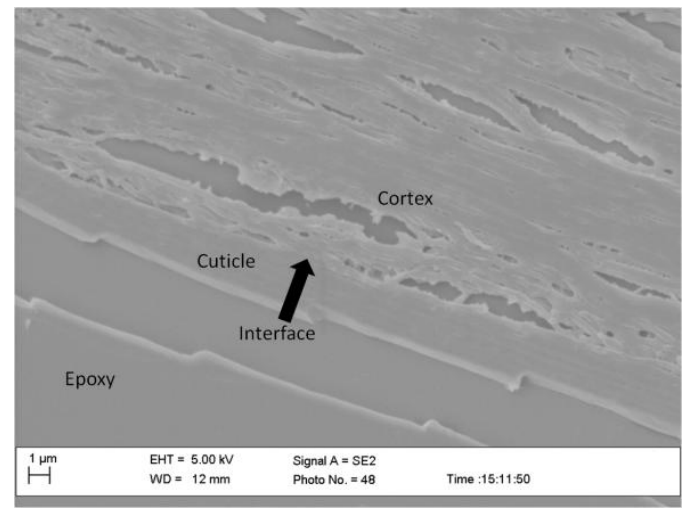

Figure 10. SEM image of the cuticle-cortex (black arrow) interface for the hair shown in Fig. 7. appear to be affected. 

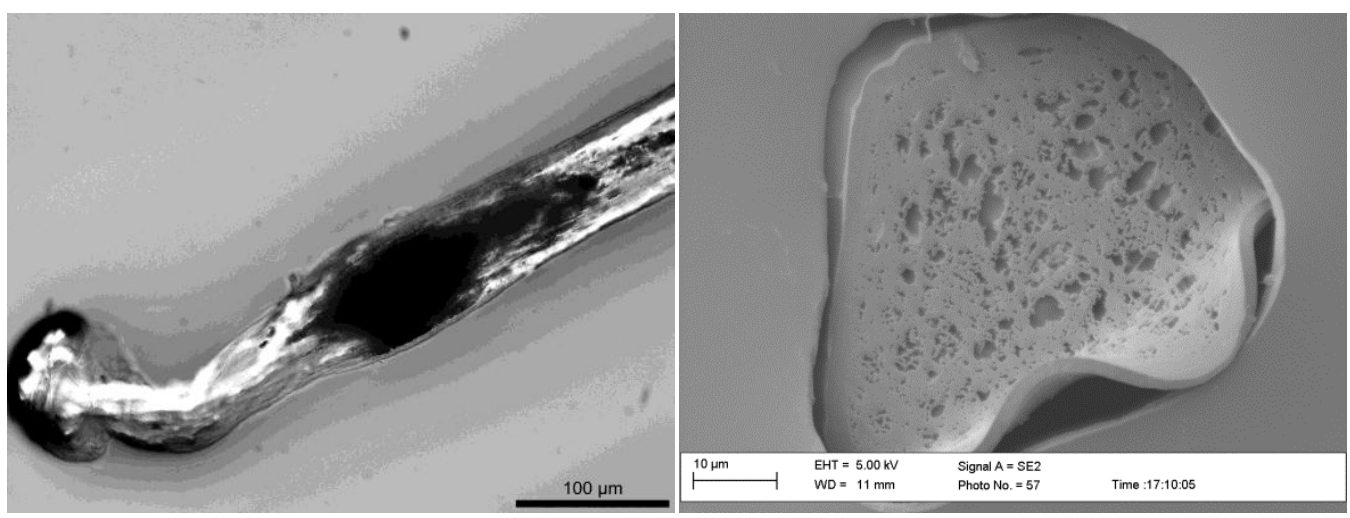

399 Figure 11. Light micrograph of a hair displaying a known PMRB (left) and SEM image (right) of the 400 corresponding cross-section. Note that the damage to the hair is randomly distributed across the hair 401 section with no apparent directionality. In addition, the cuticle is unaffected by the process that produces PMRBs. Note that the hair section has pulled away from the epoxy and has slightly wrinkled. 
407 [1] Petraco N, Fraas C, Callery FX, Forest PRD. The morphology and evidential significance of human hair roots. J Forensic Sci. 408 1988;33:68-76.

409 [2] State of Florida v. Casey Marie Anthony. 2008.

410 [3] Kogut v. Nassau County New York. 2012.

411 [4] Koch SL, Michaud AL, Mikell CE. Taphonomy of Hair-A study of postmortem root banding. J Forensic Sci. 2013:s52-s9.

412 [5] Gaudette BD. Evidential value of human hair examination. In: Robertson J, editor. Forensic examination of hair. London:

413 Taylor and Francis; 1999.

414 [6] Bisbing RE. The Forensic Identification and Association of Human Hair. In: Saferstein R, editor. Forensic Science Handbook.

415 Englewood Cliffs, New Jersey, USA: Prentice Hall Regents; 1982. p. 184-221.

416 [7] Seta S, Sato H, Yoshino M, Miyasaka S. Morphological changes of hair root with the time lapsed after death. Journal of the 417 Forensic Science Society. 1984;4.

418 [8] Forensic Human hair examinatin guidelines. Scientific Working Group on Materials Analysis (SWGMAT); 2005.

419 [9] Tafaro JT. The use of microscopic postmortem changes in anagen hair roots to associate questioned hairs with known hairs 420 and reconstruct events in two murder cases. J Forensic Sci. 2000;45:495-9.

421 [10] Kadane J. Post-mortem root banding of hairs: a sceptical review. Law, Probability and Risk. 2015;0:1-16.

422 [11] Popescu C, Hocker H. Hair-the most sophisticated biological composite material. Chem Soc Rev. 2007;36:1282-91.

423 [12] Lee YJ, Rice RH, Lee YM. Proteome analysis of human hair shaft. Molecular \& cellular proteomics 55. 2006;5:789-800.

424 [13] Rice RH. Proteomic analysis of hair shaft and nail plate. J Cosmet Sci. 2011;62:229-36.

425 [14] Birbeck MSC, Mercer EH. The electron microscopy of the human hair follicle: Part 1. Introdcution and the hair cortex. The 426 Journal of biophysical and biochemical cytology. 1957;3:2013-214.

427 [15] Birbeck MSC, Mercer EH. The electron microscopy of the human hair follicle: Part 2. The hair cuticle. The Journal of 428 biophysical and biochemical cytology. 1957;3:215-22.

429 [16] Birbeck MSC, Mercer EH. The electron microscopy of the human hair follicle: Part 3. The inner root sheath and 430 trichohyaline. The journal of biophysical and biochemical cytology. 1957;3:223-30.

431 [17] Swift JA. The electron histochemistry of cystine-containing proteins in thin transverse sections of human hair. Journal of 432 the Royal microscopical society. 1968;88:449-60.

433 [18] Swift JA, Bews B. The chemistry of human hair cuticle- II: The isolation and amino acid analysis of the cell membranes and 434 A-layer. J Soc Cosmet Chem. 1974;25:355-66.

435 [19] Swift JA, Bews B. The chemistry of human hair cuticle-III: The isolation and amino acid analysis of various subfractions of 436 the cuticle obtained by pronase and trypsin digestion. J Soc Cosmet Chem. 1976;27:289-300.

437 [20] Baden HP. Hair Keratin. In: Orfanos CE, Happle R, editors. Hair and Hair Diseases: Springer-Verlag; 1990.

438 [21] Lever WF, Schaumberug-Lever G. Histopathology of the Skin. Philadelphia, PA: J.B. Lippincott Company; 1990.

439 [22] Yu J, Yu D-w, Checkla, Daniel M., Freedberg IM, Bertolino AP. Human hair keratins. Journal of Investigative Dermatology.

440 1993;101:56s-9s.

441 [23] Swift JA, Bews B. The chemistry of human hair cuticle-I: A new method for physical isolation of cuticle. J Soc Cosmet Chem.

$442 \quad 1974 ; 25: 13-22$.

443 [24] Swift JA. The Hair surface. In: Orfanos CE, Stuttgen WMG, editors. Hair Reseach Status and Future Aspectes. Berlin-

444 Heidelberg: Springer-Verlag; 1981. p. 712. 
[25] Forslind B. The growing anagen hair. In: Orfanos CE, Happle R, editors. Hair and Hair Diseases. Berlin- Heidelberg: Springer446 Verlag; 1990. p. 1057.

447 [26] Wortmann FJ, Wortmann G, Zahn H. Pathways for dye diffusion in wool fibers. Journal of textile research. 1997;67:720-4.

448 [27] Robbins CR. The morphology and chemistry of human hair. Proceedings of the International Symposium on Forensic Hair

449 Comparisons. Washington, DC: Federal Bureau of Investigation, U.S. Government Printing Office; 1985. p. 3-21.

450 [28] Harding H, Rodgers G. Physiology and growth of human hair. In: Robertson J, editor. The Forensic Examination of Hair.

451 London, United Kingdom: Taylor \& Francis; 1999.

452 [29] Paus R, Cotsarelis G. The biology of hair follicles. The New England Journal of Medicine. 1999;341:491-7.

453 [30] Higgins CA, Westgate GE, Jahoda CAB. From telogen to exogen: mechanisms underlying formation and subsequent loss of 454 the hair club fiber. Journal of Investigative Dermatology. 2009;129:2100-8.

455 [31] Linch CA, Prahlow JA. Postmortem microscopic changes observed at the human head hair proximal end. J Forensic Sci 456 2001;46:15-20.

457 [32] Collins BW. The effect of temperature and environment on post mortem morphology of human hair roots. New York:

458 Thesis: John Jay College of Criminal Justice; 1996.

459 [33] Delgado RJ. An investigation to replicate post mortem characteristics in ante mortem anagen head hair. Thesis: California 460 State Univesity, Los Angeles; 2013.

461 [34] Domzalski AC. The effects of environmental exposure on human scalp hair root morphology. New York: Thesis: John Jay 462 College of Criminal Justice; 2004.

463 [35] Linch CA, Smith SL, Pralow JA. Evaluation of the human hair root for DNA typing subsequent microscopic comparison. J

464 Forensic Sci. 1998;43:305-14.

465 [36] Daubert v. Merrell Dow Pharmaceutical. 1993.

466 [37] Frye v. U.S.

467 [38] Collier JH. Estimating the postmortem interval in forensic cases through the analysis of postmortem deterioration of 468 human hair. Thesis- Louisiana State University; 2005.

469 [39] Shaw S. The microscopic characteristics of antemortem and postmortem hairs at the root end. Proceedings of the 470 American Academy of Forensic Sciences. Atlanta: Victor Graphics, Inc.; 2012. p. 116-7.

$471[40]$ Swift JA. The electron histochemial demonstration of cystine-containing proteins in the Guinea pig hair follicle.

472 Histochemie. 1969;19:88-98.

473 [41] Happey F, Johnson AG. Some electron microscope observations of hardening in the human hair follicle. J Ultrastructure 474 research. 1962;7:316-27.

475 [42] Harland DP, Vernon JA, Walls RJ, Woods JL. Transmission electorn microscopy staining methods for the cortex of human 476 hair: a modified osmium method and comparison with other stains. Journal of Microscopy. 2011;243:184-96.

477 [43] Kassenbeck P. Morphology and Fine Structure of Hair. In: C.E. Orfanos WM, and G. Stuttgen, editor. Hair Research Status 478 and Future Aspects. Berlin: Springer-Verlag; 1981. p. 52-64.

479 [44] Kaszynski E. Hair Growth: Mechansim and regulation. Proceedings of the International Symposium on Forensic Hair 480 Comparisons. Washingto, DC: Federal Bureau of Investigation, U.S. Government Printing Office; 1985. p. 23-33.

481 [45] Chang BS, Hong WS, Lee E, Yeo SM, Bang IS, Chung YH, et al. Ultramicroscopic observations on morphological changes in 482 hair during 25 years of weathering. Forensic Sci Int. 2005;151:193-200.

483 [46] DeGaetano DH, Kempton JB, Rowe WF. Fungal tunneling of hair from buried body. J Forensic Sci. 1992;37:1048-54. 
[47] Schaefer H, T.E.Redelmeier. Skin barrier. Principles of Percutaneous Absorption. Basel: Karger; 1996. 